\title{
AJKFluids2015-15294
}

\section{NUMERICAL AND EXPERIMENTAL STUDY ON NEGATIVE BUOYANCE INDUCED VORTICES IN N-BUTANE JET FLAMES}

\author{
Yuan Xiong \\ Clean Combustion Research \\ Center, KAUST \\ Thuwal, Saudi Arabia
}

\author{
Min Suk Cha \\ Clean Combustion Research \\ Center, KAUST \\ Thuwal, Saudi Arabia
}

\author{
Suk Ho Chung \\ Clean Combustion Research \\ Center, KAUST \\ Thuwal, Saudi Arabia
}

\begin{abstract}
Near nozzle flow field in flickering n-butane diffusion jet flames was investigated with a special focus on transient flow patterns of negative buoyance induced vortices. The flow structures were obtained through Mie scattering imaging with seed particles in a fuel stream using continuous-wave (CW) Argon-ion laser. Velocity fields were also quantified with particle mage velocimetry (PIV) system having $\mathrm{kHz}$ repetition rate. The results showed that the dynamic motion of negative buoyance induced vortices near the nozzle exit was coupled strongly with a flame flickering instability. Typically during the flame flickering, the negative buoyant vortices oscillated at the flickering frequency. The vortices were distorted by the flickering motion and exhibited complicated transient vortical patterns, such as tilting and stretching. Numerical simulations were also implemented based on an open source $\mathrm{C}++$ package, LaminarSMOKE, for further validations.
\end{abstract}

\section{NOMENCLATURE}

$D \quad$ Inner diameter of the jet nozzle

$\mathrm{Fr} \quad$ Froude number defined as $\mathrm{Fr}=U_{\text {jet }} / \sqrt{g D}$

$g \quad$ Gravitational acceleration, $9.8 \mathrm{~m} / \mathrm{s}^{2}$

$U_{j e t} \quad$ Mean velocity of the fuel jet

\section{INTRODUCTION}

Flickering laminar nonpremixed flames have been widely encountered in daily lives such as flickering candle flames and the dancing flairs of wood fires. Practically, flickering flames can either enhance the generation of soot particles that facilitates a heat transfer process in industrial boilers [1-3] or causes a combustion instability coupled with pressure fluctuation [4-6]. In this regard, it is necessary to understand the flow structure of flickering flames to design a more stable combustion system.

Depending on the density of fuel relative to ambient air, buoyancy acting on the fuel can be either positive, such as methane, or negative, such as n-butane [7]. The flow structure of positive buoyant laminar nonpremixed jet flames have been extensively studied both numerically and experimentally and relatively well understood [8-11]. For example with a small Froude number, Fr, of methane flame, varicose vortical structure, located in the oxidizer side of the jet flames, can be visualized using optical techniques [9, 12]. Observed flickering flames with bare eyes are induced by a formation of vortices. As Fr increases, relatively small and fast rolling-up vortices can be identified in the fuel side of a jet flame due to a shear layer instability [9].

Negative buoyant fuels, such as LPG and vaporized liquid fuels, are more widely used in industrial combustors. Comparing to positive buoyant fuels, another vortical structure in a fuel core region have been reported regardless of Fr [7, 8, 11].

For small Fr with steady flames of negative buoyancy, due to the appearance of the vortex in a fuel core region, a negative axial velocity was found at a nozzle exit. Therefore, setting an inlet boundary condition with a predetermined shape such as a parabolic or uniform profile at a nozzle exit was not appropriate in numerical simulations [7].

For large $\mathrm{Fr}$ with flame flickering motions, more complicated vortical structure in a fuel core region could be observed [7]. However, the interaction between the flickering dynamics and negative buoyancy driven vortical structures in the fuel core is yet to be discovered. 
In this study, a transient vortical structure coupling with a flame flickering motion was investigated experimentally and numerically. Mie scattering was used to visualize the vortical structure. Quantitative velocity fields were obtained via $\mathrm{kHz}$ PIV. Numerical simulations were also conducted using an open source code LaminarSmoke [13] under OpenFOAM [14] framework.

\section{EXPERIMENT}

A coflow burner consisted of a central fuel nozzle and surrounding coflowing air section was used. A stainless steel tube with an inner diameter of $10.8 \mathrm{~mm}$ and a thickness of 1.0 $\mathrm{mm}$ was used as the fuel nozzle. The length of the nozzle was $855 \mathrm{~mm}$ to guarantee the fuel flow to be fully developed in the laminar regime. The coflow air section consisted of the layer of plastic beads and a ceramic honeycomb to ensure a uniform velocity profile.

Mass flow controllers were used to control the flow rates. N-butane (>99.5\%), which is heaviest among gaseous fuels under the standard thermodynamic condition, was used as fuel. Compressed air was used for the coflow, and its velocity was set at $6.2 \mathrm{~cm} / \mathrm{s}$. In a previous study it has been shown that a nozzle heating can affect a flow structure [7]. To exclude this effect, all measurements have been conducted within 10s after igniting flames, thus the temperature of the fuel nozzle can be treated as a room temperature.

An Ar-ion laser (Spectra-Physics, Stabilite 2017) at 488 nm with $4 \mathrm{~W}$ was used to illuminate seed $\mathrm{TiO}_{2}$ particles $(\sim 1$ $\mu \mathrm{m})$ to visualize the flow patterns of the fuel jets. A set of micro-lenses (Leica, Z16 APO) was used for magnification with a digital camera. Particle image velocimetry was adopted to measure a velocity field with a $1 \mathrm{kHz}$ repetition rate. A time interval between two consecutive images was set to $500 \mu$ s. Lavision Davis 8.1 was used to analyze the velocity data. The experimental setup is shown in Fig.1 schematically.

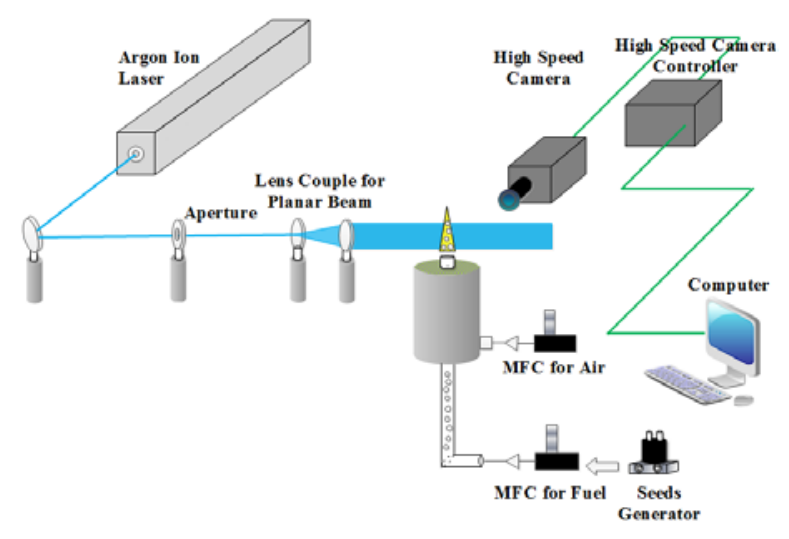

FIGURE 1. Schematic of experimental setup

\section{SIMULATIONS}

LaminarSmoke code developed under the framework of OpenFOAM was used to validate experimental results [15-18].
Operator splitting technique was adopted to separate stiff chemical reactions from non-stiff hydrodynamic processes. Governing equations were based on continuous, multicomponent, compressible and thermally-perfect mixtures of gases. Details on the equations could be found in [18]. Temporal and spatial discretization has been implemented within OpenFOAM framework, thus it was not elaborated here. Computational domains and associated boundary conditions were shown schematically in Fig.2.

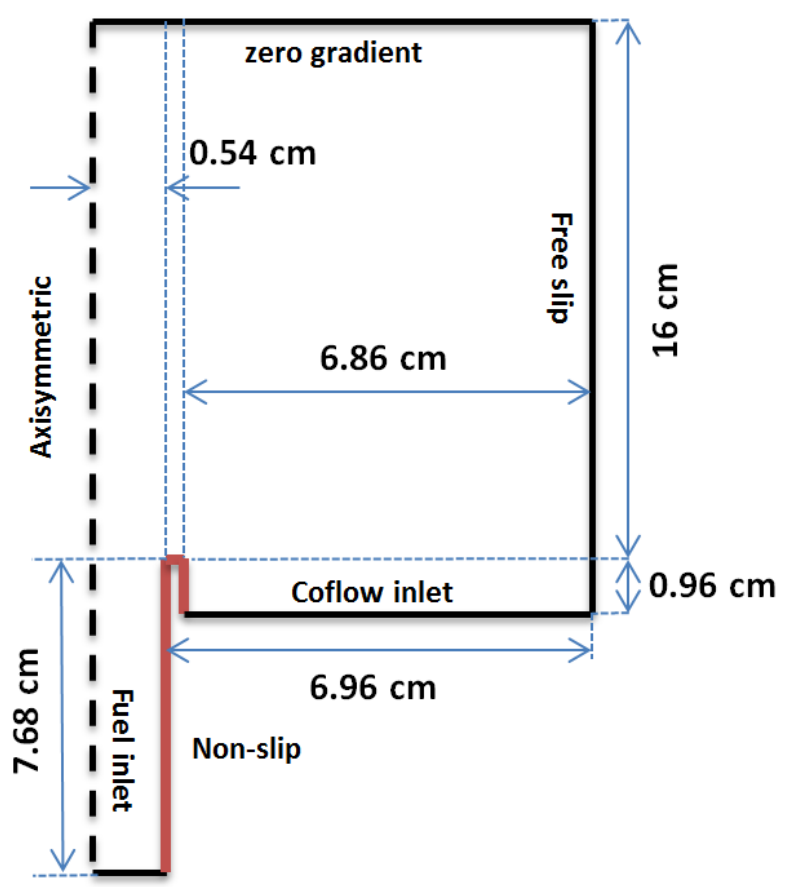

FIGURE 2. Schematic of computational domain setup

Multi-block strategy, dividing the computational domain into four sections, was adopted to specify the exact location of the nozzle and also to save the amount of required grids. Grid independence and domain size sensitivity were tested, and resulted domain size is marked in Fig. 2 with a total number of grids with 42,240 .

\section{MIE SCATTERING ANALYSIS}

The Mie scattering images of a vortical structure of nbutane jet flames are shown in Fig. 3. Those images were selected within a period of flame flickering, and the corresponding mean velocity of fuel at the exit of the nozzle was $6.22 \mathrm{~cm} / \mathrm{s}$. The fuel velocity was selected to be similar with the coflow velocity to minimize a potential contribution from coflow velocity to the observed vortice formation [8].

A relatively large-sized toroidal vortex can be clearly identified about $1 \mathrm{~cm}$ above the nozzle exit. As a contrasting result from the steady vortex with small Fr cases in the previous study [7], here the vortex exhibits transient behavior coupled with the flame flickering motion. The complicated vortical structure fluctuated, while it synchronized with the flame 
flickering motion. The size and shape of the vortical structure also changed significantly during the fluctuation.

Two magnified images of both the minimized and maximized size of the vortex have been shown in Fig.4. The cross-sectional area of the vortex can differ by near factor of two as marked with the yellow dashed lines. The sudden expansion of the vortex shown in Fig.4 is due to the outward movement flame front driven under the bulge vortices in the oxidizer side, which initiates the flame flickering.

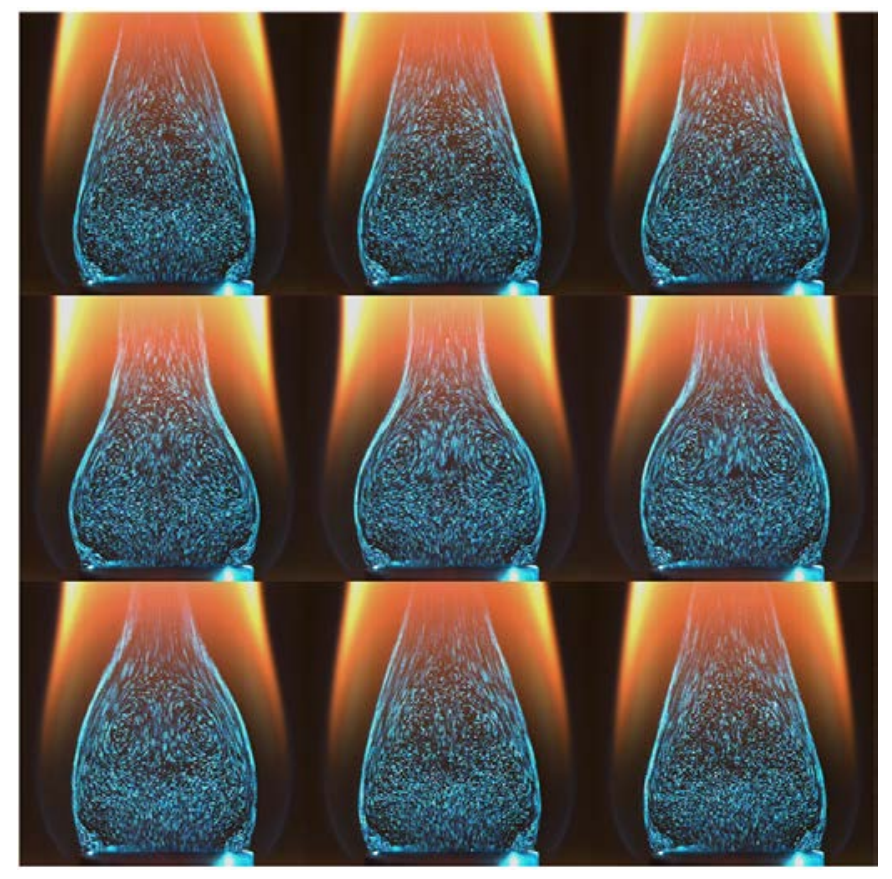

FIGURE 3. Negative buoyance driven vortical structure in the near nozzle area coupled with flame flickering motions in one period while flame flickering frequency is about $12 \mathrm{~Hz}$.

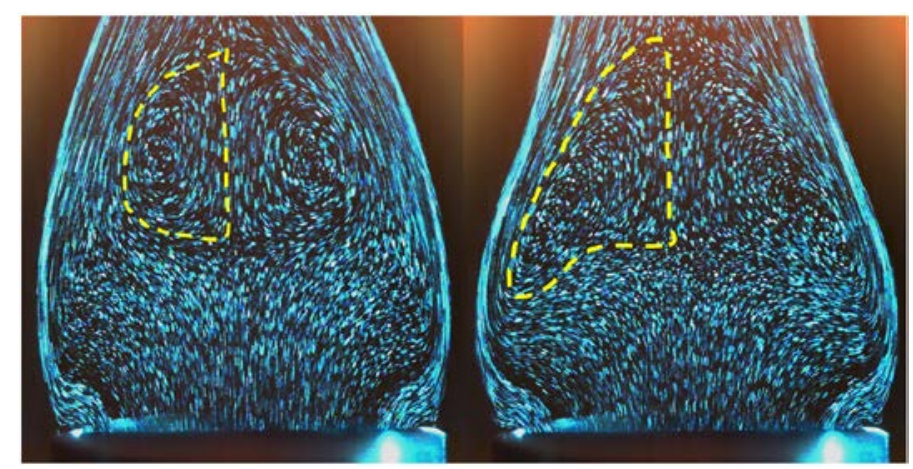

FIGURE 4. Minimized and maximized negative buoyant vortical structure in the near nozzle area due to flame flickers

\section{PIV ANALYSIS}

Due to the expansion and contraction process of the vortical structure shown in Fig. 4, a surrounding velocity field is expected to be modified during a period of flickering. To measure the velocity field, PIV at $1 \mathrm{kHz}$ repetition rate was utilized. Time interval between the recorded images is set at $500 \mu$ s to allow better resolution over the low velocity inner vortical region, instead of a fast moving velocity field near the flame zone. Obtained velocity field is shown in Fig. 5. A uniform length of vectors was used and color code represents the magnitude of the velocity as shown in the figure. Similar to Fig. 3, the instantaneous velocity fields were selected during one period of flickering.

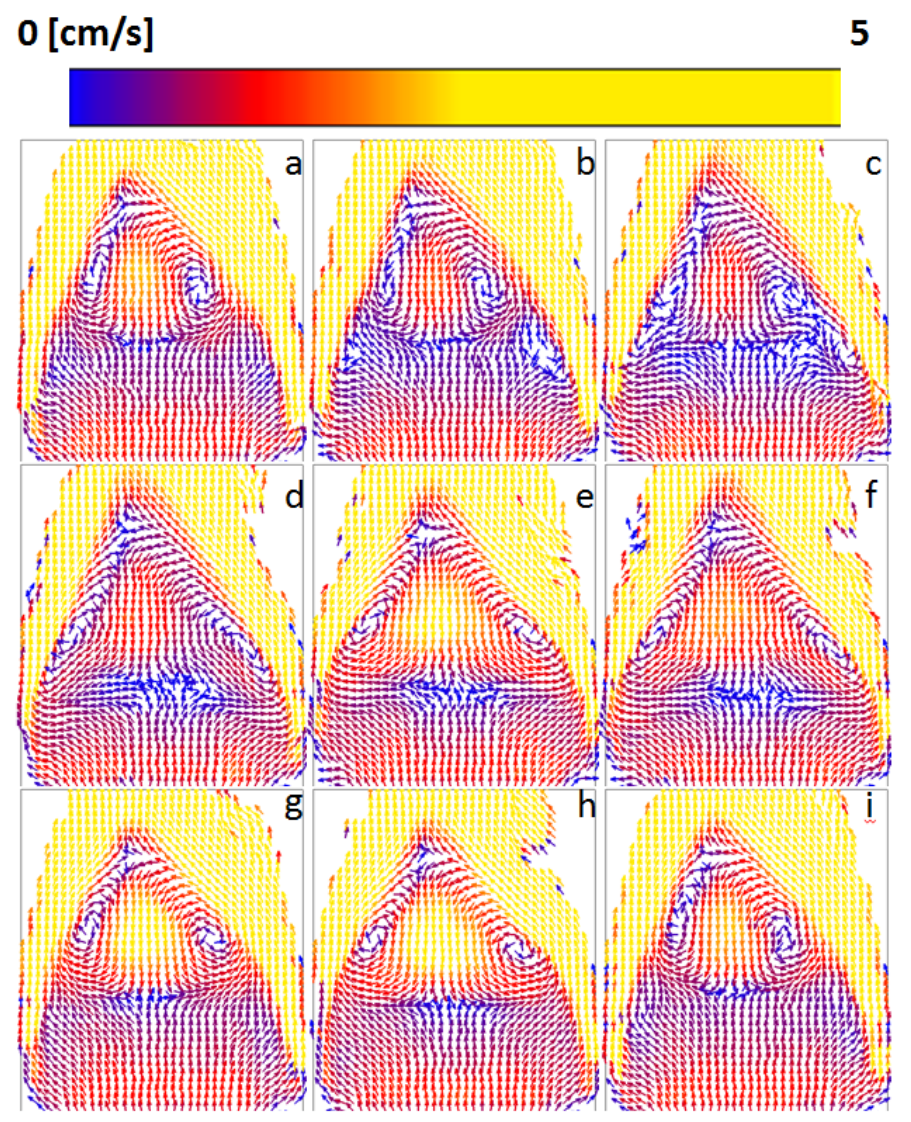

FIGURE 5. Temporal resolved vortical structure in the near nozzle area coupled with flame flickers in one period.

Note that color code ranged from 0 to $5 \mathrm{~cm} / \mathrm{s}$ to focus on the low velocity vortical region. It is noted that when the size of vortical structure is small as shown in Fig. 5a, all upstream velocity vectors point downstream indicating compressing effect of the vortical structure due to the neighboring flow fields. However, in Fig. 5b and 5c, it can be noted that due to the expansion of the flame front to the radial direction at the flame bottom, there is a reversed flow near the wings of the high velocity layer colored as yellow. As a result, the vortex extends into this reverse flow region and merges it together, which resulted in the increases in its size as shown in Fig. 5d, e, and $\mathrm{f}$. This is a main process for the doubling of the crosssectional area of the vortex as observed in Fig. 4. As the flame 
front moves again back to the center of the jet, the upstream flow field of the vortical structure starts to accelerate and push the edge of the vortical structure downstream. Due to the surrounding high-speed flow field near the flame front, the vortical size needs to shrink again.

\section{NUMERICAL SIMULATIONS}

It has been proved that certain depth inside the nozzle needs to be included in the computational domain to accurately simulate negative buoyancy flames [7]. Thus, inlet boundary condition was specified at $7.68 \mathrm{~cm}$ down from the nozzle exit in the simulations. Calculated results with the experimental conditions were plotted in Fig. 6. Streamlines and temperature profile indicating the location of reaction zone are co-presented.

The transient vortical behavior was successfully predicted as shown. Similar variation of the vortical structure to the results in Figs 3-5 was found. In addition, a yellow dashed reference lines were also plotted and a more converged flame surface can be noted in Fig. 6h compared to the case in Fig. 6e, which explains the accelerated flow field and consequent shrinking of the vortical structure.

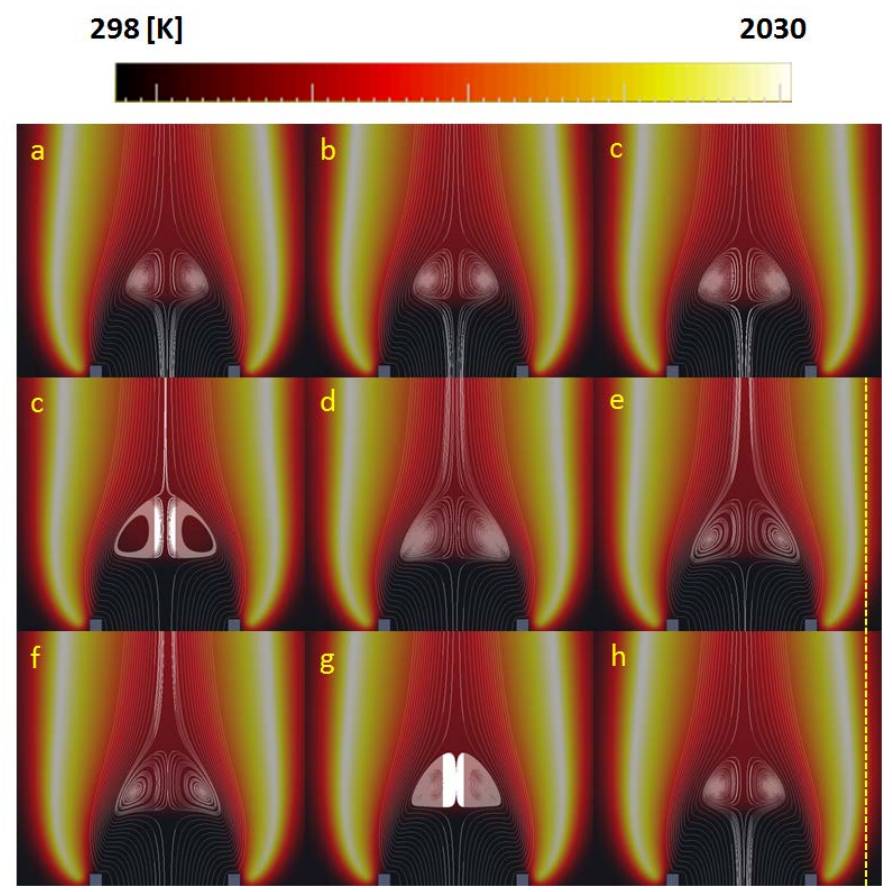

FIGURE 6. Simulated temporal resolved vortical structure in the near nozzle area coupled with flame flickers in one period.

\section{CONCLUSIONS}

The transient vortical structure induced by the negative buoyancy acting on the fuel was studied with moderate Froude number with flame flickering. Diagnostic techniques, such as Mie scattering and PIV, were applied and numerical simulation was also implemented to validate the experimental results. The vortical structure was strongly coupled with the flickering motion. Therefore, the size of the vortical structure varied significantly during a period of flame flickering. PIV measurement showed that the variation of the vortical structure was mainly due to the velocity field surround the vortical structure. Numerical simulations well predicted the experimental observations.

\section{ACKNOWLEDGMENTS}

Research reported in this publication was supported by Competitive Research Funding from King Abdullah University of Science and Technology (KAUST).

\section{REFERENCES}

[1] Smyth, K.C., et al., 1993. "Greatly enhanced soot scattering in flickering $\mathrm{CH}$ 4/air diffusion flames”. Combustion and flame, 95(1): pp. 229-239.

[2] Shaddix, C.R., Harrington, J.E., and Smyth, K.C., 1994. "Quantitative measurements of enhanced soot production in a flickering methane/air diffusion flame”. Combustion and Flame, 99(3): pp. 723-732.

[3] Kaplan, C.R., Shaddix, C.R., and Smyth, K.C., 1996. "Computations of enhanced soot production in time-varying CH 4/air diffusion flames”. Combustion and Flame, 106(4): pp. 392-405.

[4] Lang, W., Poinsot, T., and Candel, S., 1987. “Active control of combustion instability”. Combustion and Flame, 70(3): pp. 281-289.

[5] McManus, K., Poinsot, T., and Candel, S., 1993. “A review of active control of combustion instabilities". Progress in energy and combustion science, 19(1): pp. 1-29.

[6] Lieuwen, T.C. and Yang, V., 2005. "Combustion instabilities in gas turbine engines(operational experience, fundamental mechanisms and modeling)". Progress in astronautics and aeronautics.

[7] Xiong, Y., Cha, M.S., and Chung, S.H., 2015. "Fuel density effect on near nozzle flow field in small laminar coflow diffusion flames”. Proceedings of the Combustion Institute, 35(1): pp. 873-880.

[8] Chen, L.D. and Roquemore, W.M., 1986. "Visualization of jet flames”. Combustion and Flame, 66(1): pp. 81-86.

[9] Chen, L.D., et al., 1989. "Buoyant diffusion flames". Symposium (International) on Combustion, 22(1): pp. 677684.

[10] Davis, R.W., et al., 1991. "Preliminary results of a numerical-experimental study of the dynamic structure of a buoyant jet diffusion flame”. Combustion and Flame, 83(34): pp. 263-270.

[11] Chen, L.D., et al., 1992. "Time evolution of a buoyant jet diffusion flame”. Symposium (International) on Combustion, 24(1): pp. 303-310.

[12] Gotoda, H., Kawaguchi, S., and Saso, Y., 2008. "Experiments on dynamical motion of buoyancy-induced flame instability under different oxygen concentration in 
ambient gas”. Experimental Thermal and Fluid Science, 32(8): pp. 1759-1765.

[13] Cuoci, A., et al., 2013. "A computational tool for the detailed kinetic modeling of laminar flames: Application to C2H4/CH4 coflow flames". Combustion and Flame, 160(5): pp. 870-886.

[14] Jasak, H., Jemcov, A., and Tukovic, Z., 2013. "OpenFOAM: A C++ library for complex physics simulations".

[15] Jin, H., et al., 2015. "Experimental and kinetic modeling study of laminar coflow diffusion methane flames doped with 2-butanol”. Proceedings of the Combustion Institute, 35(1): pp. 863-871.

[16] Jin, H., et al., 2014. "Experimental and kinetic modeling study of PAH formation in methane coflow diffusion flames doped with n-butanol”. Combustion and Flame, 161(3): pp. 657-670.

[17] Cuoci, A., et al., 2013. "Numerical Modeling of NOx Formation in Turbulent Flames Using a Kinetic Postprocessing Technique”. Energy \& Fuels, 27(2): pp. 11041122. 